\section{Potential Adverse Interactions Between Allopathic Drugs, Herbals and Dietary Products: Mechanisms of Action and Clinical Implications}

\author{
Ginpreet Kaur ${ }^{1 *}$ and Harpal S Buttar ${ }^{2}$ \\ ${ }^{1}$ Department of Pharmacology, SPP School of Pharmacy \& Technology \\ Management, SVKM's NMIMS, Mumbai, Maharashtra, India \\ ${ }^{2}$ Department of Pathology \& Laboratory Medicine, University of Ottawa, \\ Ontario, Canada
}

\section{Introduction}

In the beginning of the $19^{\text {th }}$ century, allopathic or synthetic medicines became the primary mode of treatment. Fortunately in the $21^{\text {th }}$ century, there are many choices for treating communicable and non-communicable diseases with synthetic pharmaceuticals, alternative remedies, Natural Health Products (NHPs) and dietary supplements. Inspite of the enormously rapid development of new drug discoveries made in the field of allopathy, plant-derived products, Ayurvedic remedies, and Chinese medicines still remain the major sources of therapy in the developing countries. Self-therapy with nutraceuticals is also becoming popular globally among the lay population. According to the WHO statistics of 2008, around $80 \%$ population in developing countries relies on traditional plant-derived remedies, and such traditional medicines make around $25 \%$ market share of the entire pharmacotherapy cache [1].

With the changes in modern lifestyle, sedentary habits, and excessive availability of fast foods/drinks loaded with large amounts of sugar, the incidences of obesity and type 2 diabetes as well as cardiovascular and musculoskeletal diseases have escalated in both developed and developing countries. Multiple drug therapy is often needed for treating the cardiovascular diseases and the co-morbid chronic conditions, especially in the elderly population. Overwhelming majority of presently used synthetic drugs are metabolised by $\mathrm{CYP}_{450} 3 \mathrm{~A} 4$ and $\mathrm{CYP}_{450} 2 \mathrm{D} 6$.

${ }^{*}$ Corresponding author: Ginpreet Kaur, Department of Pharmacology, SPP School of Pharmacy \& Technology Management, SVKM's NMIMS, VL Mehta Road, Vile Parle, Mumbai, Maharashtra, India, Tel: +91 42332000, Extn: 2035; E-mail: ginpreet.aneja@gmail.com

Citation: Kaur G, Buttar HS (2016) Potential Adverse Interactions Between Allopathic Drugs, Herbals and Dietary Products: Mechanisms of Action and Clinical Implications. J Diabetes Metab Disord 3: 009.

Received: February 22, 2016; Accepted: February 27, 2016; Published: March 14, 2016

Copyright: (c) 2016 Kaur G and Buttar HS, This is an open-access article distributed under the terms of the Creative Commons Attribution License, which permits unrestricted use, distribution, and reproduction in any medium, provided the original author and source are credited.
There is a global concern about the adverse effects of drug-drug, drug-herbal and drug-food interactions because many serious, and occasionally life-threatening, adverse consequences have occurred in humans when allopathic drugs are taken along with some herbal remedies, foods, fruit juices, and dietary supplements. Herbal medicines include single or multiple herbs, crude herbal preparations and finished herbal products, which contain plant-derived biologically active ingredients [2].

As mentioned earlier, the usages of herbal remedies and dietary supplements have increased in developed and developing countries due to the belief of absence of side effects and this practice has led to interactions of synthetic drugs with plant-based medicines and fruit juices. Many patients tend to self-treat their medical conditions with herbal medicines along with allopathic drugs, resulting in drug-herbal/food interaction. Mostly, the possibility of herb-drug-diet interactions had been ignored during the revival of plant-derived medicinal use [3]. Several well designed clinical studies have shown adverse effects following the simultaneous oral intake of a wide variety of synthetic drugs with certain natural health products, such as grapefruit or pomegranate juice, and St. John's wort. The combined oral administration of grapefruit juice and St. John's wort can alter the metabolic disposition and bioavailability of a wide variety of synthetic drugs and consequently affect their safety and efficacy in patients.

The interactive effects may be additive, synergistic or antagonistic in nature, and may complicate the dosing regimen of long-term medications, or lead to undesirable side effects. The possibilities of herb-drug or food-drug interactions increase as the number of medications being used increase, subsequently increasing the chances of undesirable Adverse Drug Reactions (ADRs). Clinically relevant pharmacokinetic and pharmacodynamic drug-herbal/food interactions usually occur due to the alteration in absorption, distribution, metabolism and elimination of allopathic drugs. Such ADRs may not be seen when the allopathic drugs are administered individually.

Drug-herb/diet interactions may be significantly important for drugs with a narrow therapeutic range (e.g., warfarin, digoxin, antiarrhythmics), and for sensitive populations such as elderly patients, pregnant and nursing mothers as well as very sick and frail individuals (AIDS and cancer patients) who may be exposed to polypharmacy $[4,5]$. Multiple ingredients in herbs, food and dietary supplements may modify the intestinal $\mathrm{pH}$ or motility, inhibit or induce gut transporters (P-glycoprotein) or metabolizing enzymes $\left(\mathrm{CYP}_{450}\right.$-isozymes), glucuronidation pathway, and thus change the rate and extent of absorption, metabolism and disposition of synthetic drugs.

The quality, purity and potency of herbal medicines are not generally regulated by many drug regulatory agencies as they are done for the synthetic drugs. Few examples of the major herb-drug interaction include: Prolongation of clotting time in patients taking warfarin along with garlic (Allium sativum), giving rise to bleeding complications. Furanocoumarins (paradisins, bergamotin) present in grapefruit juice modify the metabolism of alprazolam and felodipine by inhibiting the activity of $\mathrm{CYP}_{450} 3 \mathrm{~A} 4$ and Pgp transporters in the 
gastrointestinal tract and liver [6]. The main biologically active ingredients (hypericin, hyperforin) of St. John's wort, a popular herbal medicine used for treating mild depression, anxiety and insomnia, are known to elevate the action of antidepressants, especially SSRI type of drugs (e.g., paroxetine). Coriander (Coriandrum sativum) enhances the effects of hypoglycaemic agents [7].

In summary, herb-drug and diet-drug interactions are considered one of the leading causes of patient morbidity and mortality. Before prescribing an allopathic medication, physicians should enquire whether their patients are consuming an interacting diet or herbal product and either instruct their patients to stop consuming such products or adjust dosage to compensate for drug-diet or drug-herbal effects [8]. Collaborative efforts are required from patients, physicians, and pharmacists as well as industry (drug manufacturers, suppliers of herbals, food, and nutraceuticals) to minimize or possibly prevent any potential risks associated with the concomitant use of natural health products, herbal remedies, dietary supplements and interacting allopathic drugs.

This commentary is meant to highlight the mechanisms of drug-herb/food interactions, clinical outcomes, and the regulatory initiatives (post-market surveillance and labelling changes) being taken by regulatory agencies in Canada, America, Europe, Japan and Australia to meet the challenges associated with rapidly emerging area of drug-herb-diet interactions. Hopefully, Drug \& Food Regulators and physicians in Asia and other developing countries will also pay attention to potential ADRs and clinical implications that can occur by the combined oral administration of allopathic drugs, herbals and dietary products [9].

\section{References}

1. WHO (2004) WHO Guidelines on Safety Monitoring of Herbal Medicines in Pharmacovigilance Systems. World Health Organization, Geneva, Switzerland.

2. Mukherjee PW (2002) Quality Control of Herbal Drugs: An Approach to Evaluation of Botanicals. Business Horizons Publishers, New Delhi, India.

3. Izzo AA, Ernst E (2009) Interactions between herbal medicines and prescribed drugs: an updated systematic review. Drugs 69: 1777-1798.

4. Wehling M. (2009): Multimorbidity and polypharmacy: how to reduce the harmful drug load and yet add needed drugs in the elderly? Proposal of a new drug classification: fit for the aged. J Am Geriatr Soc,57: 560-1

5. Ginpreet Kaur (2013): Polypharmacy: The past, present and the future, Journal of Advanced Pharmaceutical Technology \& Research,4(4):224-225

6. Bailey D, Malcolm J, Arnold O, David Spence J (2002) Grapefruit juice-drug interactions. Br J Clin Pharmacol 46: 101-110.

7. Ayo JA, Agu H, Madaki I (2005) Food and drug interactions: its side effects. Nutrition \& Food Science 35: 243-252.

8. Eidi M, Eidi A, Saeidi A, Molanaei S, Sadeghipour A, et al. (2009): Effect of coriander seed (Coriandrum sativum L.) ethanol extract on insulin release from pancreatic beta cells in streptozotocin-induced diabetic rats. Phytother Res 23: 404-406.

9. Bailey DG, Dresser G, Arnold MO (2013) Grapefruit-medication interactions: Forbidden fruit or avoidable consequences? CMAJ 185: 309-316. 